\title{
Atomic-resolution STEM-EELS to probe and stabilize superconductivity in thin films
}

\section{Berit Goodge and Lena Kourkoutis}

\section{School of Applied and Engineering Physics, Cornell University, United States}

Quantum materials provide opportunities for realizing and combining functionalities not available in conventional materials such as multiferroicity, colossal magnetoresistance, topological protection, and high-temperature superconductivity. In this broad class of materials, quantum phenomena often emerge at atomic length scales due to strong interactions between charge, spins, and the lattice. These interactions also afford various tuning knobs such as light, strain, and external fields which may be used to further control and manipulate such properties for applications. Understanding the nature of these interactions, the relevant length scales at which quantum phenomena arise, and the role of disorder requires advanced spatially resolved probes.

Modern scanning transmission electron microscopes (STEM) provide the spatial resolution to map the atomic lattice structure of quantum materials. Nevertheless, not all defects or inhomogeneities critical to the material's behavior are easily identified using high-resolution imaging alone. In these cases, localized spectroscopic measurements of the electronic states are necessary. Here, we will focus on two classes of superconducting materials, the layered perovskite ruthenates and the recently reported infinite-layer nickelates [1].

Nickelate superconductors have, thus far, only been successfully synthesized in thin film form where the substrate is believed to provide necessary structural stabilization. Furthermore, complete phase purity in the reduced infinite-layer films has not been achieved yet. Accessing the electronic states of the superconducting state without contributions from unreduced regions of the film requires spatially localized spectroscopic probes. An additional challenge of the material's metastable nature is their sensitivity to electron dose as well as the low cross-sections of the relevant EELS edges, requiring extensive acquisition times and therefore excellent stage and instrument stability. With the combination of an atomic-scale STEM probe and high energy resolution $(\delta \mathrm{E}<0.4 \mathrm{eV})$, localized EELS measurements revealed not only the Mott-Hubbard nature of the infinite-layer $\mathrm{NdNiO}_{2}$ compound, but also the multiband contributions from $\mathrm{O} 2 p, \mathrm{Ni} 3 d$, and $\mathrm{Nd} 5 d$ states [2].

Not all superconductors are as robust to structural inhomogeneities as the infinite-layer nickelates. For example, the more well-known superconducting ruthenates are extremely sensitive to disorder and point defects such as site vacancies are known to play a key role but have been challenging to quantify [3]. Such point defects are perhaps the most extreme version of localized inhomogeneities, and can mask the functional or exotic properties such as superconductivity. Even when $\mathrm{Sr}_{2} \mathrm{RuO}_{4}$ thin films appear structurally pristine by most metrics (including X-ray powder diffraction and atomic-resolution HAADFSTEM imaging), some are found not to undergo a superconducting transition while others exhibit some of the highest reported $\mathrm{T}_{\mathrm{c}} \mathrm{s}$ [4]. We will demonstrate how high-resolution STEM-EELS enables not only identification but also quantification of Ru vacancies in the non-superconducting films. These microscopic 
insights are critical to building reproducible quantum material platforms and understanding how novel properties can be stabilized.

This work was supported by AFOSR (FA 9550-16-1-0305) and NSF (DMR-1539918, DMR-1719875, DMR-1429155).

\section{References}

[1] D. Li et al., Nature 572 (2019) p. 624.

[2] B.H. Goodge et al., Proc. Nat. Acad. Sci. 118, e2007683118 (2021).

[3] G. Kim et al., Phys. Rev. Mat. 3, 094802 (2019).

[4] H.P. Nair et al., APL Materials 6, 101108 (2018). 